\title{
El proceso de escritura científica en la formación doctoral: una aproximación narrativa'
}

\author{
The Scientific Writing Process in Doctoral Training - \\ A Narrative Approach \\ MARISOL REY CASTILLO \\ Tecnológico de Monterrey \\ México \\ marisol.rey@teachers.org
}

(Recibido: IO-O2-2O2I; aceptado: $05^{-0} 5^{-202 \mathrm{I})}$

Resumen. El desarrollo de competencias investigativas es el centro de la formación doctoral. La escritura de textos científicos es uno de los aspectos del desarrollo de dichas competencias. Esto abarca no solamente los aspectos lingüísticos o formales de la escritura del texto, sino también el proceso de publicación en una revista científica. Durante la formación doctoral los estudiantes deben aprender sobre esos procesos, a partir de distintas actividades o tareas, generalmente guiadas por sus maestros y tutores. El estudio tuvo el objetivo de identificar las estrategias adoptadas por los doctorandos en los procesos de escritura y publicación de un artículo científico, y lo que esto significó para ellos en su proceso de formación doctoral. Se eligió el estudio de caso, mediante el enfoque cualitativo narrativo, en el que dos doctorandos relataron la experiencia de escritura y publicación de uno de sus artículos. Como resultados principales destacan la investigación documental como estrategia importante para iniciar en la escritura científica, el acompañamiento de las tutoras de tesis, no solo como literacy brokers sino también como coautoras, el cuidado en el proceso editorial para lograr la publicación, la relevancia de la producción escrita como acto de construcción de conocimiento útil para los demás y la valoración que se da al proceso como parte esencial en la formación como doctores.

Palabras clave: formación doctoral; escritura científica; proceso de escritura.

\footnotetext{
${ }^{\text {I }}$ Para citar este artículo: Rey Castillo, Marisol (2022). El proceso de escritura científica en la formación doctoral: una aproximación narrativa. Alabe 25. [www.revistaalabe.com]

DOI: IO.I5645/Alabe2O22.25.7
} 
Abstract.The development of research competencies is the center of doctoral education.Writing scientific texts is one of the aspects of the development of these competences. This involves not only the linguistic aspects of writing, but also the process of publishing in a scientific journal. During doctoral education, students learn about these processes, based on different activities or tasks, generally guided by their advisors and tutors. This study aimed to identify the strategies used by doctoral students in the writing and publishing processes of a scientific article, and what this meant for them in their doctoral education process. The methodology was case study, using the narrative qualitative approach, in which two doctoral students reported the experience of writing and publishing one of their articles. The main results highlight that documentary research stands out as an important strategy to start in scientific writing, as well as the accompaniment of thesis tutors, not only as literacy brokers but also as co-authors. Other aspects, like the editorial process to achieve publication, the relevance of written production as an act of construction of useful knowledge for others, and the assessment that is given to the process as an essential part in the training of doctors, were also important.

Keywords: PhD education; scientific writing; writing process. 


\section{Introducción}

\section{I.I. La escritura científica}

Una investigación no estará debidamente finalizada en tanto no se han comunicado sus resultados ante la comunidad científica y en general ante quienes puedan estar interesados en la información que proporciona un nuevo conocimiento (García Del Junco \& Castellanos Verdugo, 2007; Gastel \& Day, 20I6; Rogers, 2003). El texto científico es un tipo de documento académico que reporta los resultados de una investigación con unas características específicas que permite identificar no solamente los marcos conceptuales, sino también la metodología seguida, los hallazgos y las conclusiones a las que se ha llegado. Este se enmarca dentro de un proceso de escritura científica que sirve como medio de comunicación en la ciencia para entregar a la comunidad el nuevo conocimiento logrado a partir de procesos propios de investigación, por lo cual debe tener características como la claridad, la coherencia y la precisión (Gastel \& Day, 2or6; MacKenzie, 20I5; O’Connor, 2003; Singh \& Mayer, 2014).

El proceso de escritura científica requiere el seguimiento de una serie de acciones formales, no solamente relacionadas con aspectos netamente textuales de redacción o verbalización del conocimiento, sino procesos de pensamiento mayores que implican la organización del contenido de manera clara, coherente y pertinente para la exposición de aquello que se quiere comunicar, por lo cual existe un conjunto de habilidades y operaciones cognitivas que se ponen en juego antes, durante y después de la construcción del texto, evidenciados en la planeación, la escritura y la corrección (Flower \& Hayes, I996; Gastel \& Day, 20I6; Hayes, I996; Hayes \& Flower, I980; Radloff \& De La Harpe, 200o).

Dado que el texto científico tiene características convencionales aceptadas por la comunidad académica, quien realiza un documento como producto de una investigación debe seguir una serie de patrones para ofrecer los resultados y que estos sean aceptados por los evaluadores y el editor de una revista para su posterior publicación y que implica la presentación de unos marcos conceptuales, la explicación de la metodología empleada, los resultados o hallazgos y las conclusiones finales como parte de aporte al conocimiento. El proceso editorial exige adherirse a estas formas particulares para que el texto pueda ser ofrecido ante los lectores (Gastel \& Day, 2oI6; O’Connor, 2003; Parija \& Kate, 2OI7).

\section{I.2. La escritura científica y la formación de investigadores}

La formación doctoral tiene como eje fundamental el desarrollo de competencias investigativas para que los educandos puedan desempeñarse en su futuro profesional como investigadores, con todos los elementos que esto implica. Uno de estos elementos es la capacidad de poder comunicar ante la comunidad académica los hallazgos de las investigaciones, de tal manera que parte de la formación en un doctorado es el desarrollo de competencias para la producción y difusión del texto científico (Carrasco Altamirano et al., 2O2O; Carrera Hernández et al., 2OI7; Castro Azuara \& Sánchez Camargo, 20I6; 
Garcés-Prettel \& Santoya-Montes, 20I3; Moreno Bayardo, 20II, 2009). Para ello, los programas doctorales adoptan distintos tipos de acciones curriculares que incluyen cursos, seminarios, talleres, investigación que lleva a la escritura de una tesis, entre otros. Los profesores y tutores emplean distintas actividades didácticas y formativas, individuales y grupales, que no solamente incluyen la investigación, sino también la comunicación de los hallazgos de la misma con el fin de que los estudiantes puedan desarrollar las competencias necesarias en el ejercicio formativo, tal y como se ha demostrado en estudios previos (Bandenhorst \& Guerin, 20I6; Emig et al., 20I6; Fernández Fastuca \& Wainerman, 20I5; Lam et al., 20I9; Lei \& Hu, 20I9; Martínez Amilpa, 2or8; Moreno Bayardo, 2007; Saeed et al., 20I9; Wisker, 2015).

Por un lado, se encuentran aquellas estrategias propiamente textuales que permiten la escritura propiamente dicha del documento, para las cuales el estudiante recibe formación a partir de distintas actividades en su programa: cursos, seminarios, talleres, charlas, instrucción directa del asesor. Por otro lado, se encuentran aquellas estrategias en las que se realizan actividades que van más allá de proporcionar directrices o indicaciones, e involucran al doctorando en una actividad de escritura, ya sea de manera individual, o de manera grupal con otros estudiantes, o también acompañado por sus asesores o tutores, que toman el papel de literacy brokers (Jerskey, 20I3; Lillis \& Curry, 2006) entendidos éstos como aquellas personas que realizan un acompañamiento al proceso de construcción del texto, más allá de la revisión final del documento y que abarca tareas relacionadas con la forma lingüística, pero también con el contenido del texto, por lo cual ha de ser una persona de la misma área de conocimiento (Carrasco Altamirano et al., 202O; Jerskey, 20I3; Lam et al., 20I9).

De igual manera, el proceso de publicación se involucra la comunicación con los gatekeepers, o grupo de personas que se constituyen como autoridad en la revista y cuya misión va más allá de identificar los aspectos textuales de calidad para que el texto pueda ser efectivamente publicado, y se convierten en personas que enriquecen el texto a través de la retroalimentación previa a la publicación (Curry \& Lillis, 2OI4). Para llevar a cabo este proceso, también es necesario contar con una serie de estrategias que posibiliten llevar cumplir con el propósito de lograr la publicación. Adicionalmente, el proceso editorial entraña distintos pasos, desde la misma selección de la revista, y otras condiciones como las líneas temáticas de la revista, el alcance, la difusión, incluso el tipo de artículos que publica, y que los autores deben tener en cuenta para lograr la publicación (Gastel \& Day, 20I6).

En términos generales, este tipo de estrategias adoptadas por los doctorandos tanto en la producción escrita como en el proceso de publicación han sido abordadas no solo desde lo teórico, sino también desde la perspectiva del dos tutores o asesores (Carrasco Altamirano et al., 2020; Fernández Fastuca \& Wainerman, 2OI5; Lam et al., 20I9; Matarese, 20I2; Quiles-Fernández et al., 20I8). Sin embargo, en pocas ocasiones se analizan estos fenómenos desde la voz del doctorando, por lo cual es válido analizar el proceso desde el ángulo de quien es el centro del proceso formativo y así determinar el 
camino que lleva su experiencia de aprendizaje no solamente desde las actividades propuestas por los profesores, sino también desde sus propios procesos reflexivos, desde sus necesidades, sus acciones, sus aprendizajes y la manera en la que el proceso de escritura hace parte de la formación como investigador.

El propósito de este trabajo fue identificar las estrategias adoptadas por los doctorandos en los procesos de escritura y publicación de un artículo científico, producido en el contexto de su formación como doctores educativos. Esto permitirá tener una perspectiva del proceso que siguieron, así de lo que significó para ellos dentro de su formación doctoral, y de los aprendizajes y reflexiones a las que llegaron a través de ese proceso. Las estrategias, aprendizajes y reflexiones relatadas por los estudiantes, pueden constituirse como un ejemplo para otros estudiantes de doctorado en contextos similares, y también como reflexión para los tutores a fin de fortalecer y afianzar estos procesos en sus alumnos.

\section{Método}

\section{I. Diseño y participantes}

Se eligió estudio de caso, mediante el enfoque cualitativo narrativo (Chase, 2OI7; Creswell \& Poth, 20I8; Czarniawska, 2004) el cual se vale de las narraciones de uno o dos informantes quienes cuentan sus historias, entregándolas individualmente, utilizando un orden cronológico que permite ordenar el significado de la experiencia o vivencia narrada (Clandinin \& Caine, 20I6; Coulter \& Smith, 2009; Creswell \& Poth, 2OI8). En este caso particular, la historia contada giró alrededor de la escritura y proceso editorial de un artículo científico publicado en una revista de alto impacto, y todo lo que se relaciona con este, que permitió identificar elementos propios del acto de escribir en este nivel académico, como parte de la formación como investigador en su programa doctoral. La preferencia por lo narrativo se da porque se quiere tener una perspectiva general de la escritura y la publicación como procesos anclados a la formación de doctores.

Teniendo en cuenta que el enfoque posibilita la participación de pocos individuos (Chase, 20I7; Creswell \& Poth, 20I8; Czarniawska, 2004), para este estudio se analizaron las narrativas de dos informantes: Alejandro y Liliana ${ }^{2}$. Cada uno de los informantes narró la historia de uno de sus artículos, por separado, en una interacción con el investigador, presentando un relato cronológico del proceso que siguió uno de sus artículos científicos publicados en una revista de alto impacto, desde que nació la idea de realizar la investigación que devino en el texto, hasta que vieron el artículo publicado en la revista. La elaboración de ese artículo se enmarca en su proceso de formación como doctores, y que es parte de las actividades de su formación. Al momento de realizar el artículo, Liliana se encontraba cursando materias de cuarto y quinto semestre, mientras que Alejandro estaba en segundo, y en ambos casos la escritura del texto es parte de sus actividades formativas.

\footnotetext{
${ }^{2}$ Pseudónimos.
} 
El doctorado que cursaban los participantes es en el área de la educación, cuya titulación puede optar por cuatro líneas de investigación: tecnología educativa, psicopedagogía, educación en ciencias y educación, sociedad y cultura. Este es ofertado por una universidad privada ubicada en una de las ciudades más pobladas del norte de México. La modalidad es presencial y la duración es de cuatro años: los dos primeros destinados a cursar distintas asignaturas en el campo de la educación y la metodología de la investigación, y los dos últimos enfocados exclusivamente al trabajo en la tesis doctoral, bajo la tutela de un asesor (aunque desde el primer semestre se inicia el desarrollo de la tesis). Entre varios requisitos de graduación, destaca el tener una producción académica de por lo menos tres artículos publicados en revistas indexadas en Scopus, y que se encuentren en los tres primeros cuartiles del SCImago Journal Rank (SJR). Así, escribir y publicar se convierte en un aspecto necesario para los estudiantes de este programa. A pesar de este requisito, el plan de estudios no tiene ninguna asignatura, seminario, o taller dirigido a orientar a los estudiantes en el proceso de escritura científica, sino que las competencias escriturales se desarrollan de manera transversal en las asignaturas relacionadas con metodología de la investigación, y en la construcción de la tesis doctoral.

Al momento de realizar la investigación, el doctorado contaba con diecisiete estudiantes activos, distribuidos en los ocho semestres; entre estos, once ya habían logrado publicar por lo menos un artículo. Para este trabajo se eligió a dos estudiantes que se constituyeran como una muestra la población de estudiantes que se encuentran en formación y que ya habían logrado publicar, es decir que pudieran identificarse como dos casos típicos para ilustrar al grupo en general. La elección se hizo al azar, de la cual resultaron Alejandro y Liliana quienes aceptaron participar.

\subsection{Instrumentos}

Con respecto a los instrumentos, para este caso se eligieron dos: entrevista a los participantes, y además se analizaron fuentes documentales, específicamente, los borradores del artículo producido, así como el artículo final publicado y el intercambio de corespondencia con el editor de la revista y/o las rúbricas de evaluación por parte de los árbitros del artículo biografías (Chase, 20I7; Creswell \& Poth, 20I8; Czarniawska, 2004; Given, 2OI2).

De acuerdo con Given (2OI2), la entrevista narrativa debe estar organizada para facilitar el desarrollo de la narración. En ese sentido, más que tener una entrevista semiestructurada con preguntas plenamente establecidas, se optó por una única pregunta inicial en la que se pudiera plantear los lineamientos para que el informante iniciará con su relato. A medida de que este iba avanzando, surgían en la interacción nuevas preguntas que permitían profundizar en algunos elementos del relato u orientar la narración (Creswell \& Poth, 20I8; Czarniawska, 2004; Wortham, 2OOI). La pregunta inicial solicitaba al participante narrar el proceso de escritura del artículo en cuestión, desde que se gestó la idea de la investigación que devino en el artículo, hasta que lo vio publicado.

Los borradores del texto y el documento final permitieron identificar la progre- 
sión del artículo y evidenciar el relato cronológico compartido por Liliana y Alejandro. Así como se observaron los borradores del artículo, también fue relevante revisar el diálogo entre el autor y el árbitro de su artículo o el editor de la revista que le transmite el mensaje de retroalimentación sobre el escrito. Esto posibilitó el reconocimiento de la dinámica que tuvo este autor con un externo, en este caso la revista, y cómo se integró la retroalimentación de los evaluadores al documento y con esto al proceso de la escritura.

\subsection{Procedimientos}

Para la recolección de datos se citó a los estudiantes por separado, se les formuló la misma pregunta inicial y se grabó la narrativa en audio. En ambos casos fue necesario realizar preguntas adicionales, que fueron distintas dependiendo de lo que surgió en cada narrativa. Además, los informantes hicieron llegar, a través de correo electrónico, los borradores de los escritos y el intercambio comunicativo con el editor y árbitros del artículo. Las entrevistas y los documentos para el análisis se consolidaron en dos archivos de audio (que fueron transcritos), diecinueve borradores de artículos, dos artículos finales y doce formatos o correos de intercambio del proceso de evaluación editorial.

Estos treinta y cinco archivos fueron puestos en el software ATLAS.ti versión 7.5.4. para facilitar el proceso de análisis. Se crearon los códigos a partir de la información extraída de cada uno; dichos códigos fueron agrupados en cinco familias que generaron las categorías: contexto de la producción escrita, proceso de elaboración del artículo, trabajo con coautores, proceso editorial y escritura científica en el proceso de formación doctoral. Estas se señalaron en el texto generando 96 citas extraídas de los documentos. El proceso metodológico del trabajo se realizó en cinco fases que abarcaron desde el cuestionamiento inicial hasta el análisis de datos. La Figura i muestra de forma concreta los pasos seguidos para la investigación.

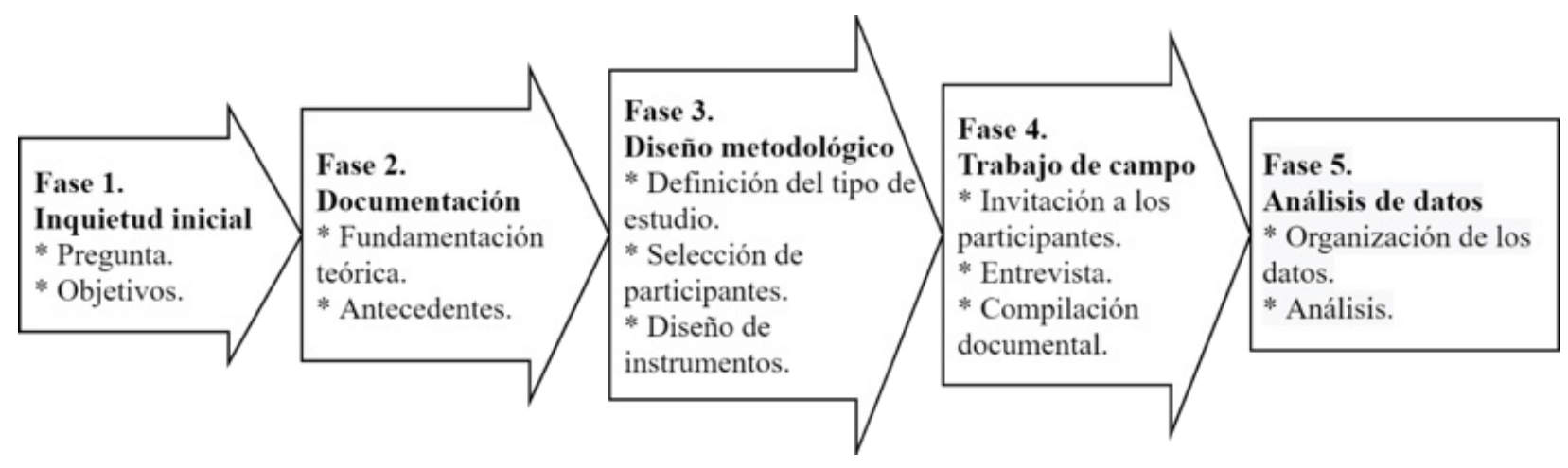

Figura I. Fases de la investigación. 


\section{Resultados}

A partir de los datos se han identificado cinco elementos característicos en el proceso de escritura de los dos doctorandos en este caso. Estos no solo tienen relación con el texto escrito, sino que involucran otros aspectos del proceso como el contexto de la producción, la necesidad que motiva a la publicación, el trabajo colaborativo, el proceso editorial y el aprendizaje adquirido como investigadores en formación. En los subapartados a continuación se presentan estos.

\section{I. Contexto de la producción}

En ambos casos se escribe el texto como producto de una investigación documental, específicamente un mapeo sistemático de literatura y una revisión sistemática de literatura. Específicamente en ese doctorado, realizar mapeos es una estrategia para identificación de posibles problemas de investigación. Ese es el caso de Alejandro, cuya revisión se realiza por recomendación de su directora de tesis como parte del proceso de documentación e identificación de posibles problemas relevantes para la investigación de su tesis. Después de ese mapeo, surge la idea de publicarlo como resultado de la investigación documental:

Cuando empecé el doctorado todavía no estaba muy enfocado en el tema, entonces, junto con mi asesora, ella me propuso hacer un mapeo para identificar los posibles temas [...] y ya después decidimos que podía publicarse como un mapeo sistemático de literatura (Entrevista a Alejandro).

Con respecto a Liliana, la revisión sistemática de literatura nace a partir de una idea que le surge gracias a una actividad que realiza en su función como coordinadora editorial de una de las revistas de la institución. A pesar de que esta actividad no está ligada a su proceso de investigación para la tesis ni a ninguno de los cursos que toma, considera que el trabajo anexo que hace en la revista es parte fundamental de su formación doctoral y como parte de los requisitos para obtener el título, y que realizar una revisión sistemática en ese contexto le ayudará a afianzar sus habilidades investigativas:

Tuve la oportunidad de que me incluyeran como coordinadora editorial en la revista (...) La idea fue buscar estrategias de difusión de la revista y aprovechar esa información para un artículo, como un producto académico que, además de ser parte de mi formación en el doctorado, es requisito. (Entrevista a Liliana).

Dada la naturaleza de estas investigaciones de tipo documental, el proceso en ambos casos fue de aproximadamente seis meses, desde que se inició la investigación hasta que se vio publicado el artículo al final. Alejandro comenzó el proceso de investigación y escritura del artículo en el primer semestre de sus estudios, mientras que Liliana ya se 
encontraba en quinto semestre:

Pues la idea fue a comienzo de agosto, cuando comencé el doctorado

(...) en marzo ya entré a la revista y ahí estaba publicado (Entrevista a Alejandro).

Este tipo de investigaciones documentales para identificar problemáticas en un campo de conocimiento suele ser común en un proceso de formación doctoral, como se ha documentado en otros estudios (Quiles-Fernández et al., 2OI8; Wisker, 2OI5).

\subsection{Proceso de elaboración del artículo}

Partiendo del contexto descrito anteriormente, el proceso de elaboración del escrito comenzó, en ambos casos, después de toda la indagación documental seguida en el proceso de investigación. Los estudiantes señalan que iniciaron el artículo por la construcción de la fundamentación teórica, seguida por la redacción de los fundamentos metodológicos, los resultados y las conclusiones, por lo cual se puede identificar que la manera en la que escriben el artículo respeta la misma estructura que lleva el documento final, lo cual sigue lo recomendado desde la teoría (Gastel \& Day, 2oı6; Hayes \& Flower, I980; Radloff \& De La Harpe, 2000):

Para esto empecé a leer algunos artículos como para ir escribiendo una conceptualización teórica de lo que se trataba este tema (...) el artículo fue una planeación de la metodología que se iba a seguir. En la metodología de este tipo de temas, o de artículo hay que planear unos pasos como muy específicos y ya hay autores que marcan una metodología bien clara (...) Cuando ya había, ya estaba la base de datos completa, pues se empezó la redacción (Entrevista a Alejandro).

Esto no solamente es dicho por los alumnos, sino también se encuentra evidenciado a partir de los distintos borradores del texto; entre uno y otro denotan el avance del escrito, en los que se puede identificar la progresión paulatina que sigue el documento en su construcción.

De igual manera, los dos alumnos coinciden en que la sección más compleja para ellos fue la construcción de la fundamentación teórica, pues requirió una indagación mayor que llevó más tiempo que el resto de las secciones, en la que el discurso pudo construirse de una manera más fluida a partir de sus propios procedimientos de investigación y de los hallazgos que realizaron durante el proceso, aspecto que también ha sido documentado desde la teoría (Flower \& Hayes, r996; Hayes, r996; Hayes \& Flower, r98o):

Lo que más demora es el inicio, porque hay que buscar las referencias del marco teórico del trabajo y pues eso, hay que buscar y redactar que quede coherente y que sí vaya a servir para dar sustento que es un poco complicado (Entrevista a Liliana). 
Otro aspecto en el que los dos estudiantes coinciden es la consulta de otros escritos similares como modelo para la escritura, estrategia que es usada con frecuencia en el proceso de construcción de textos (Matarese, 2OI2; Saeed et al., 2OI9):

También leyendo otras revisiones y viendo, tratando de aplicar lo que otros hacen al propio trabajo (Entrevista a Alejandro).

Alejandro afirma que algunos aspectos de la redacción la resultaron un tanto complejos, por lo cual solicitaba a su coautora el acompañamiento en esas secciones específicas:

Ya cuando veía que algo me quedaba mal o estaba inseguro, pues ya le decía a mi asesora y ella me ayudaba y lo escribía o me decía cómo escribir (Entrevista a Alejandro).

Liliana narra que antes de iniciar la escritura en pleno de las secciones, realizó un esbozo de lo que contendría cada una de las partes para facilitar el proceso de escritura y para orientarla a ella y a sus coautores en la redacción del texto, lo cual es recomendado desde la teoría (Flower \& Hayes, I98I; Hayes \& Flower, r980):

Antes de la redacción hice como un esquema de lo que iba en cada parte y así tener una forma de saber, de guiarnos en la escritura y que fuera más rápido (Entrevista a Liliana).

Los dos estudiantes mencionan la necesidad de la constante lectura, corrección y trabajo en los borradores que no se evidencia solamente como avances progresivos del texto, sino también como la relectura de las secciones y la articulación de las mismas para reflejar una coherencia plena del texto (Gastel \& Day, 20I6; Hayes \& Flower, I980).

\subsection{Trabajo con coautores}

Tanto Alejandro como Liliana realizaron y publicaron el artículo en coautoría. En el caso de Alejandro, la coautora fue su directora de tesis; Liliana contó no solamente con su directora de tesis, sino también con otra de las estudiantes del doctorado que junto con ella se desempeña como coordinador editorial en la misma revista científica. (Quiles-Fernández et al., 20I8).

En ambos casos se puede identificar la coautoría de las asesoras de ambos estudiantes. Estos roles tomaron un papel doble. El primero, durante el proceso de investigación, desde la dirección y evaluación del procedimiento como tal, mientras que los autores principales conducían el proceso investigativo propiamente dicho. En la formación de la escritura académica, tener un tutor de escritura o literacy broker se ha constituido como esencial en el proceso, porque su función no es únicamente valorar el texto terminado, sino orientar progresivamente en la construcción del mismo (Jerskey, 2OI3; Lillis \& Curry, 2006; Martinez \& Graf, 20I6):

Yo iba haciendo la búsqueda, ella me iba diciendo y me corregía cuando 
yo le presentaba los avances de la base de datos (Entrevista a Alejandro).

Confrontar los resultados obtenidos de las diferentes fuentes (de acuerdo a los criterios establecidos en la metodología) e interpretarlos, con base en el marco teórico establecido; para sacar a la luz los hallazgos más significativos del estudio realizado (Nota al margen de página hecha por la coautora de Alejandro. Borrador \# 7 ).

El segundo, durante el proceso de redacción del texto, como autores propiamente dichos, construyendo conjuntamente con el autor principal distintas secciones del documento. Esto se evidencia en los borradores del documento, en los cuales se identifican los aportes escritos por cada uno de los autores, especialmente cuando las coautoras construyen fragmentos, reformulan no modifican párrafos escritos por el autor principal y, en comentarios al margen, explican la razón por la cual realizaron la modificación. Esto deja ver que no solamente hay una construcción colaborativa entre los autores, sino que el mismo tiempo se genera una conciencia sobre el texto escrito y sobre la manera en cómo debe construirse:

El resumen lo escribo yo cuando ya esté el documento (Nota al margen de página hecha por la coautora de Alejandro. Borrador \#3).

En el caso de Liliana, también contó con la coautoría de Diana, una de sus compañeras de doctorado, que participó tanto en el proceso de investigación como en la escritura del texto. Al igual que Liliana, trabajó voluntariamente en la construcción de este trabajo en el momento en el que recibe la invitación por parte de la autora principal, pues es una actividad que no está anclada a ningún espacio curricular:

Aquí es necesario agregar referencias sobre visibilización y desarrollar más. Esto lo hace Diana. (...) Se empieza por la descripción del proceso. Esto lo hace Liliana.

(Notas al margen de página hecha por Liliana delegando a su coautora y a ella misma la elaboración de las secciones. Borrador \#3).

Con respecto a la construcción conjunta del texto, en los dos casos se puede identificar que en ningún momento se realizó la redacción grupal en tiempo real, sino que los autores se organizaban con sus respectivos coautores para construir las distintas secciones de manera asincrónica, y para retroalimentar las secciones escritas por el otro y completarlas: como en los dos ejemplos anteriores. Esto no solamente se siguió para el proceso de escritura del artículo, sino también para los ajustes en el proceso de edición, posterior a la revisión de los pares evaluadores designados por la revista:

Hola Diana. Quería solicitarte tu ayuda para revisar el abstract y la ortografía general del documento como lo indica el revisor (Mensaje de 
Liliana a su coautora para corregir el documento después de la retroalimentación de los revisores. Correo \#4).

La importancia del trabajo en coautoría y la forma en la que se organiza para el éxito del escrito ya ha sido evidenciada en otros estudios (Lam et al., 20I9).

\subsection{Proceso editorial}

El proceso editorial inició, tanto para Alejandro como para Liliana, con la elección de la revista para el envío. Alejandro afirma que esto fue cuidadoso para identificar las revistas que podrían interesarse no solamente en el tema sino también en el tipo de investigación conducida para el artículo. El artículo fue admitido en la primera revista a la cual fue enviado: una revista especializada en el tema del artículo, de alto factor de impacto (Q2 según el SJR):

Analizamos revistas que podrían interesarse por el tema y por el tipo de, o sea que admitiera mappings en sus revistas, y pues al final elegimos la que, a la que se envió (Entrevista a Alejandro).

En contraste, Liliana admite que en un primer momento eligió una revista especializada en el tema y de alto factor de impacto (Q2 según el SJR), sin seleccionar o analizar otras posibilidades. El artículo fue rechazado en este primer intento, y Liliana atribuye el rechazo a la ligereza con la que se eligió la revista, por lo cual el segundo intento se realizó en una revista que en esta ocasión fue debidamente seleccionada a partir de los intereses de la revista por el tema y por el tipo de artículo, por lo cual eligió una revista también de alto impacto (Q3 del SJR) pero con un carácter de mayor apertura hacia distintos temas que la primera. En ese segundo intento el artículo fue admitido:

Nos rechazaron el artículo porque la verdad sí, lo mandamos a una revista muy especializada, y tal vez no tomamos en cuenta que esas revistas solicitan artículos con mucho, con, muy especializados y nuestro artículo no lo era tanto. Entonces pues nos dijeron que no. Y ya miramos otras revistas, ahora sí fijándonos mejor y ya lo enviamos a una que sí nos lo aceptaron (Entrevista a Liliana).

La correcta elección de la revista es un aspecto fundamental para la aceptación de un artículo, pues no todas las revistas tienen las mismas líneas temáticas, el mismo alcance, el mismo público, incluso la misma difusión, por lo que una adecuada elección debe atender a analizar si el hallazgo de los resultados es coherente con tales aspectos mencionados (Gastel \& Day, 20I6).

En ambos casos, la aceptación o el rechazo fue comunicado junto con las observaciones efectuadas por los evaluadores; adicionalmente, se acompañaban las directrices para la corrección y segundo envío al editor. En el caso de Liliana, a pesar de que la primera revista en el que el artículo fue postulado lo rechazó, las observaciones no fueron 
del todo rechazadas, pues ella asegura que las tuvo en cuenta para mejorar el documento y enviarlo a una nueva revista:

Pues nos sirvió para ver qué dificultades tenía el artículo para mejorar porque siempre todo puede mejorar, y como las observaciones fueron tantas, pues ahí modificamos antes de enviar a la segunda revista (Entrevista a Liliana).

Tanto Alejandro como Liliana manifiestan haber corregido la mayor parte del artículo a partir de las indicaciones de los evaluadores, aunque no absolutamente todo, pues los dos sustentan que, si existen suficientes argumentos para mantener un elemento intacto en la revista, prefieren sustentarlos en una respuesta anexa para el editor:

Algunas cosas sí las corregí, y también había un formato para explicar lo que no se corregía y por qué (Entrevista a Alejandro).

Para ser honesta, pues no se corrigió todo lo que ellos querían, (...) cuando uno puede sustentar el por qué tiene en cuenta algunas cosas y otras no, pues es suficiente. Eso fue lo que hicimos, sustentar que, si cambiábamos, pues iba a perder coherencia el texto (Entrevista a Liliana).

En el caso de Alejandro, tuvo que realizar dos envíos más atendiendo a las correcciones de los evaluadores, mientras que Liliana consiguió la aceptación plena en la segunda revista después de la primera corrección. Al final del proceso los artículos fueron aceptados y posteriormente publicados.

\subsection{La escritura científica en el proceso de formación}

Los dos alumnos coinciden en que el proceso de escritura del artículo se constituyó como un logro importante en su formación doctoral, más allá de las condiciones o requisitos que solicita el programa académico. Liliana resalta la utilidad que el artículo puede tener para otras personas que tengan interés por el mismo tema, además de generar una identidad como investigadores-escritores (Lillis \& Maybin, 2OI7; Quiles-Fernández et al., 20I8; Wisker, 20I5):

El artículo le puede servir a, por ejemplo, a editores de revista que quie-

ran saber estrategias de visibilización, porque lo importante es que eso le vaya a servir a alguien (Entrevista a Liliana).

Por su parte, Alejandro señala la importancia del aprendizaje obtenido en el proceso de investigación documental y escritura:

Sobre todo, aprender a cómo se escribe un mapping, y pues los pasos, y que me va a servir mucho en el proceso de mi tesis, y que ya voy a escribir mejor en mis próximos artículos (Entrevista a Alejandro).

Además, realiza una reflexión sobre la posible utilidad del artículo para los lec- 
tores, pues no es el primer artículo que publica, y siente que una de sus publicaciones previas, aunque no se encuentra en una revista de alto impacto ni especializada, podría tener un mayor impacto en la usabilidad del conocimiento aquí expresado:

No era el primer artículo, porque ya había publicado antes en una revista de divulgación, pero me parece que ese es más útil porque le puede ayudar a más gente, no estoy seguro de si hay muchas personas interesadas en un mapping (Entrevista a Alejandro).

De igual manera, los dos expresaron la dificultad que entrañó el proceso tanto de investigación como de escritura, que les provocaban sentimientos negativos:

Pues sí era muy frustrante y estresante no poder avanzar o cuando habría que arreglar algo o corregir porque sentía que no avanzaba (Entrevista a Alejandro)

Hola Diana, lamentablemente nos rechazaron el artículo. ¿Cómo lo ves? (Correo de Liliana a su coautora al recibir noticia de la revista. Correo $\left.\#_{2}\right)$.

Sin embargo, tanto Alejandro como Liliana manifiestan un sentimiento de alegría una vez logran el objetivo de dar a conocer los resultados de sus respectivas investigaciones:

Se siente una satisfacción muy grande al ver que sí valió la pena el esfuerzo y el tiempo que se le invirtió, que fue bastante y que sí rindió frutos (Entrevista a Liliana).

\section{Discusión y conclusiones}

A partir de los resultados encontrados es posible identificar aspectos relevantes del proceso de escritura científica. En primer lugar, existe la conciencia por parte de ambos estudiantes de la importancia de comunicar los hallazgos de sus investigaciones, no solamente como un requisito formativo establecido por los lineamientos del programa, sino también como parte fundamental del proceso de aprendizaje del proceso de investigación. En ambos casos, las revisiones documentales como parte de las actividades de la formación como doctores (en un caso el trabajo de la tesis y en el otro caso una tarea asignada en su función como doctor anda) generaron datos que estos estudiantes identificaron como valiosos para realizar una publicación y comunicar a la disciplina sus hallazgos. Esta forma de generar producción textual en la formación de doctores ya ha sido relatada en trabajos previos (Quiles-Fernández et al., 20I8; Wisker, 20I5) y se utiliza como una estrategia para el desarrollo de competencias escriturales, especialmente en escenarios como el doctorado que Alejandro y Liliana cursan, en el que no existen asignaturas en el 
plan de estudios que se dediquen a la formación para la escritura científica.

$\mathrm{Al}$ mismo tiempo, en ese contexto en el que no existe un espacio formal para el desarrollo de competencias escriturales (aunque la producción textual cuente como un requisito para graduarse), otras actividades se convierten en el espacio central para el desarrollo de esas competencias. Dado que los estudiantes no han recibido instrucción formal para realizar un escrito, el mismo proceso de construcción textual se convierte en una actividad de aprendizaje en el que como investigadores-escritores, deben adoptar distintas estrategias, no solo textuales, sino también organizativas, para cumplir su propósito (Gastel \& Day, 20I6; Smirnova, 20I6). Una de ellas es orientarse leyendo artículos similares al de su propia producción, es decir otras revisiones de literatura, para identificar la estructura del texto y tener un modelo en el cual identificar pautas a seguir para la construcción de su propio texto, asunto que ha sido identificado en otros trabajos previamente realizados (Matarese, 2OI2; Saeed et al., 2OI9). Asimismo, realizar un esbozo del texto que contenga las ideas generales que debe llevar cada sección para orientar la construcción textual como tal también fue utilizada, y de hecho se recomienda desde la teoría (Flower \& Hayes, r98i; Hayes \& Flower, r980).

Otra estrategia seguida por los doctorandos, consistió en escribir el texto siguiendo el orden de las secciones tal y como aparece en el texto final: iniciando por la fundamentación teórica o introducción del artículo y finalizando en la discusión. De igual manera, la fundamentación teórica se percibió lo más complejo en el proceso, pues admiten que la indagación documental para la construcción de dicha fundamentación requiere mayor cantidad de tiempo y el uso de estrategias que permitan la construcción de esa sección a partir de conceptos y nociones ya dichos en la literatura, ante lo cual deben hacer una reelaboración de lo explicado por otros autores.

Asimismo, reconocen que la lectura y corrección permanente del texto es fundamental, no solamente para minimizar el riesgo de errores de forma, sino también para que el documento adquiera la claridad comunicativa necesaria, de acuerdo con lo que buscan expresar. La corrección como parte fundamental de la escritura también es un aspecto que ha sido delineado desde lo teórico (Gastel \& Day, 20ı6; Hayes \& Flower, I980).

Uno de los aspectos que sobresale a lo largo del proceso es la escritura colaborativa con sus coautores: en el caso de Alejandro su única coautora es su misma tutora de tesis, mientras que en el caso de Liliana tiene dos coautoras, una compañera que estudia en ese mismo doctorado y su asesora de tesis. Este asunto suele ser frecuente en la formación de postgrado (Carrasco Altamirano et al., 2O2O; Lam et al., 2OI9; Smirnova, 2OI6). Tanto para Alejandro como para Liliana la coautoría enriqueció el proceso de la escritura del texto, no solamente porque encontraron la ayuda de personas más experimentadas (especialmente en aquellas secciones o partes que consideraban más difíciles), como lo eran sus tutores de tesis, sino también porque a través de la construcción del texto y de las distintas anotaciones que realizaban los coautores para ampliar, redefinir, modificar, profundizar o concretar algunas partes del documento, se generó una conciencia más amplia 
de lo que significa escribir un artículo científico. Esto también se dio por la dinámica de escritura conjunta efectuada por los doctorandos y sus coautores, pues el trabajo nunca se realizó de manera sincrónica, sino que el escrito iba pasando por una sucesión de redacción-revisión por parte de los coautores. La escritura colaborativa en la formación doctoral ha sido abordada por estudios anteriores en los cuales se ha identificado que el aprendizaje y afianzamiento de las estrategias de escritura permiten no solamente la construcción de un texto con mejor calidad, sino que produce un aprendizaje experiencia al en los autores, que aprenden no solamente de la misma actividad de la escritura, sino de las estrategias, los modos de escritura, los modos de organización y las reflexiones de sus coautores (Carrasco Altamirano et al., 2020; Lam et al., 20I9; Smirnova, 2OI6).

Desde la teoría, las tutoras de tesis en este caso son literacy brokers (Jerskey, 20I3; Lillis \& Curry, 20o6; Martinez \& Graf, 20I6) que orientan el proceso completo desde que se está gestando incluso la investigación documental, hasta que el texto se ve finalizado, desde la temática del trabajo realizado y desde el aspecto lingüístico. Además, el grado de involucramiento de estas tutoras va más allá de ser orientadoras del trabajo desde su función de literacy brokers para convertirse en coautoras del texto, porque no solamente direccionaron el trabajo de investigación de los estudiantes para hallar los datos, sino que se convierten también en participantes activas del proceso de escritura, lo cual resulta parte del proceso formativo, asunto que se convierte en una estrategia para orientar al estudiante a partir de la colaboración activa con este, no solo desde la orientación (Smirnova, 20I6).

También hay que destacar que tanto Alejandro como Liliana se constituyen como autor principal en sus artículos, pues ha llevado a cabo el proceso de investigación documental que les permitió la escritura, y por tener un papel principal en la construcción del texto al ser quienes lideraron el proceso de escritura, construyeron la mayor cantidad parte del texto y se encargaron de todo el proceso de publicación (envío del manuscrito a la revista, comunicación con los editores, liderazgo en la corrección final para el nuevo envío a los editores). De tal manera que, en este caso, aunque existieron los coautores que cumplieron con un trabajo de escritura, el proceso seguido por Liliana y Alejandro.

El trabajo con los coautores permite aprender de la experiencia de ellos y de la construcción conjunta que puede darle al texto tener distintas miradas o varias personas acompañando o trabajando en el mismo proceso, incluso cuando no se identifica un escritura conjunta y simultánea, sino la distribución de la redacción a partir de las distintas secciones del texto. Esto permite no solamente la colaboración entre investigadores, sino también la posibilidad de aprender de los demás, y más si se trata de personas que tienen más experiencia (Carrasco Altamirano et al., 2O2O; Lam et al., 2OI9; Quiles-Fernández et al., 20I8; Smirnova, 20I6).

En el proceso de publicación, también destaca la adecuada elección de la revista. Es importante tener en cuenta que estos estudiantes deben contar con producción académica para poder obtener su título de doctor, por lo cual elegir una revista que se ajustara a los requisitos solicitados por el programa. En el ejercicio de elección de la revista los doc- 
torandos atendieron no solamente a los requisitos del programa, sino también al tipo de artículo y el área de conocimiento, lo cual determina una selección cuidadosa. En el caso de Liliana, la primera elección fue hecha sin tener en cuenta alguno de estos aspectos, por lo cual su artículo no fue aceptado, lo que la condujo a ella y a sus coautoras buscar con mayor minuciosidad. Desde la teoría, la elección de la revista se constituye como un aspecto importante en el que intervienen distintos aspectos como el alcance, el área de especialidad, el público al que se dirige, y los requisitos formales para la adecuación del texto (Gastel \& Day, 2OI6).

Por otro lado, durante el proceso editorial las observaciones recibidas por los pares evaluadores, que funcionan como gatekeepers, también fue fundamental, tanto para realizar las correcciones pertinentes al documento antes de su publicación, como para obtener un aprendizaje en el proceso de escritura. Esto se evidenció en el caso de Liliana, cuyas observaciones realizadas por el evaluador de la revista que rechazó el manuscrito, fueron tenidas en cuenta para mejorar el documento y enviarlo a otra revista en la que finalmente se dio la publicación. Por supuesto, estas correcciones se realizaron a la luz del propósito comunicativo del texto, por lo cual en algunos aspectos no se realizaron las mejoras recomendadas por los gatekeepers, pero hubo oportunidad de justificarlo, y aun así se consiguió la publicación. Esto denota que, a pesar de que se considera valiosa la perspectiva del evaluador, también existe la capacidad mantener aspectos esenciales del texto y el poder argumentar la necesidad de que así se mantenga. El hecho de que finalmente los gatekeepers admitieran dichos argumentos indica que se llega a un punto de encuentro entre autores y editores (Curry \& Lillis, 20I4).

La escritura del artículo, como parte de su proceso en su formación doctoral se constituyó como un logro importante, más allá del cumplimiento de los requisitos que exige el programa para la titulación. Por un lado, los doctorandos señalan que con sus escritos han generado un aporte al conocimiento en su campo del saber, que puede ser de interés y utilidad para otros investigadores. Por otro lado, los alumnos fueron plenamente conscientes de lo que entraña el proceso de escritura y publicación de un artículo -en el cual todavía son novatos- y que fue acompañado por coautores más experimentados, quienes acompañaron el proceso de manera activa, específicamente en aquellas partes que le generaron dificultad, y como coautores. Esto además les permitió identificar distintos elementos y aprender diferentes estrategias que le serán útiles en próximas ocasiones como los ya mencionados en párrafos anteriores.

Es destacable la satisfacción personal final que deja el proceso después de haber cumplido la misión de ver el artículo publicado, incluso cuando el proceso haya sido complejo o haya generado sentimientos de frustración. Asimismo, los autores recalcan los aprendizajes adquiridos durante el proceso y la utilidad de los nuevos conocimientos plasmados en los artículos para los potenciales lectores. Esto evidencia que al proceso de escritura científica a nivel doctoral es percibido por los alumnos como un elemento fundamental para su formación y la constitución de su ser investigador con una responsabilidad social de llevar el conocimiento a quien pueda necesitarlo (Lillis \& Maybin, 2OI7; 
Wisker, 20I5).

Identificar la manera en la que los doctorandos abordan sus primeros procesos de escritura científica durante el transcurso de su programa, posibilita reconocer la manera en la que ellos asumen la tarea de escritura y publicación, no solo desde lo lingüístico textual, sino desde la manera en la que se organizan para llevar a cabo el proceso completo, como adoptan distintas estrategias a lo largo de dicho proceso y como se apoyan en personas más expertas, como sus tutores que se convierten en coautores. Dicho reconocimiento puede constituirse como un ejemplo a seguir por otros estudiantes de doctorado en escenarios similares, en los que no existen asignaturas formales donde se instruya sobre el proceso de escritura y publicación en ciencias. Asimismo, deja ver la manera en la que los tutores pueden utilizar estrategias no solo para orientar el proceso desde afuera, sin involucrarse activamente en la construcción del texto al punto de llegar a ser coautor.

Para futuras experiencias investigativas, sería valioso identificar el punto de vista del asesor que acompaña el trabajo y que a su vez se convierte en coautor del texto, y examinar desde su perspectiva el rol que tiene como tutor en un proceso de investigación que conduce a la producción textual como un literacy broker y como un participante activo que lo conduce a escribir conjuntamente con el alumno, todo esto enmarcado en un proceso de formación doctoral. Asimismo, sería valioso profundizar en algunos de los distintos aspectos aquí encontrados, para tener más detalle del proceso y reconocer a fondo la manera en cómo se construye esas estrategias durante el proceso de escritura en su contexto de producción. 


\section{Referencias bibliográficas}

- Bandenhorst, C., \& Guerin, C. (20I6). Post/Graduate Research Literacies and Writing Pedagogies. In C. Bandenhorst \& C. Guerin (Eds.), Research Literacies and Writing Pedagogies for Masters and Doctoral Writers (Vol. 3I, Issue November 2OI5, pp. 3-28). Brill. https://doi. org/ı.II63/9789004304338_oO2

- Carrasco Altamirano, A. C., Méndez Ochaita, M. F. de M., Brambila Limón, R., Encinas Prudencio, M. T. F., \& Sánchez Hernández, V. (2020). Leer y escribir como interpretación de roles , aprender de experiencias de estudiantes de doctorado. Didac, 75, 32-39. https://didac. ibero.mx/index.php/didac/article/download/35/23

- Carrera Hernández, C., Madrigal Luna, J., \& Lara García, Y. I. (2OI7). La formación de investigadores en posgrado (Una reflexión curricular). Boletin Virtual, 6-8, 53-72. https://dialnet. unirioja.es/descarga/articulo/6245323.pdf

- Castro Azuara, M. C., \& Sánchez Camargo, M. (20I6). La formación de investigadores en el área de humanidades: Los retos de la construcción de la voz autoral en la escritura de la tesis de doctorado. Revista Signos, 49(SI), 30-5I. https://doi.org/IO.4067/So7I8o9342016000400003

- Chase, S. (20I7). Narrative Inquiry: Toward Theoretical and Methodological Maturity. In N. K. Denzin \& Y. S. Lincoln (Eds.), The Sage Handbook of Qualitative Research (pp. 946-970). SAGE Publications, Inc.

- Clandinin, J., \& Caine, V. (2OI6). Narrative Inquiry. In L. Given (Ed.), The SAGE Encyclopedia of Qualitative Research Methods Narrative Inquiry (pp. 542-545). SAGE Publications, Inc. https://doi.org/IO.4I35/978I4I2963909

- Coulter, C. a, \& Smith, M. L. (2009). Discourse on Narrative Research. Educational Researcher, 38(8), 577-590. https://doi.org/IO.3ІІ02/оOI3I89Xo9353787

- Creswell, J. W., \& Poth, C. N. (2018). Qualitative Inquiry and Research Design Choosing among Five Approaches. SAGE Publications, Inc.

- Curry, M. J., \& Lillis, T. (2OI4). Strategies and tactics in academic knowledge production by multilingual scholars. Education Policy Analysis Archives, 22(32), I-28. https://doi. org/IO.I45O7/epaa.v22n32.20I4

- Czarniawska, B. (2004). Narratives in Social Science Research. In Narratives in Social Science Research. SAGE Publications, Inc. https://doi.org/IO.4I35/978I8492095O2

-Emig, J., Composition, S. C., May, N., \& Emig, J. (20I6). Writing as a Mode of Learning Published by: National Council of Teachers of English Linked references are available 
on JSTOR for this article: hWriting as a [ Mode of Learning. 28(2), I22-128. https://doi. org/Io.2307/356095

- Fernández Fastuca, L., \& Wainerman, C. (2OI5). La dirección de tesis de doctorado: ¿una práctica pedagógica? Perfiles Educativos, 37(I48), I56-I7I. http://www.scielo.org.mx/scielo. php?script=sci_arttext\&pid=SoI85-269820I50002000IO

-Flower, L., \& Hayes, J. R. (1981). A Cognitive Process Theory of Writing. College Composition and Communication, 32(4), 365-387. https://doi.org/IO.2307/356600

- Flower, L., \& Hayes, J. R. (I996). Textos en contexto. Lectura y Vida., 2-I8. https://www.researchgate.net/profile/Linda_Flower/publication/267779388_TEXTOS_EN_CONTEXTO/links/55cb68f3o8aeb975674c7927.pdf

- Garcés-Prettel, M. E., \& Santoya-Montes, Y. E. (20I3). La formación doctoral: expectativas y retos desde el contexto colombiano. Educación y Educadores, I6(2), 283-294. https://doi. org/IO.5294/edu.20I3.I6.2.5

- García Del Junco, C. J., \& Castellanos Verdugo, C. M. (2007). La difusión de las investigaciones y el formato IMRYD: Una pesquisa a propósito de la lectura crítica de los artículos científicos. (Spanish). Acimed, I5(I), I. http://search.ebscohost.com/login.aspx?direct=true $\& \mathrm{db}=\mathrm{zbh} \& \mathrm{AN}=24 \mathrm{I} 2 \mathrm{O} 343 \&$ lang $=$ es\&site $=$ ehost-live

- Gastel, B., \& Day, R. A. (20I6). How to Write and Publish a Scientific Paper (5th ed.). Greenwood.

- Given, L. (2012). Narrative Interview. The SAGE Encyclopedia of Qualitative Research Methods, 545-546. https://doi.org/IO.4I35/978I4I29639O9.n277

- Hayes, J. R. (1996). A New Framework for Understanding Cognition and Affect in Writing. In C. Levy \& S. Ransdell (Eds.), The science of writing: Theories, methods, individual differences, and applications (pp. I-27). Lawrence Erlbaum.

- Hayes, J. R., \& Flower, L. (1980). Identifying the organization of writing processes. Cognitive Processes in Writing, 3-30.

- Jerskey, M. (20I3). Literacy brokers in the Contact Zone, Year r: The Crowded Safe House. In S. Canagarajah (Ed.), Literacy as Translingual Practice (pp. 2007-22I4). Routledge Taylor \& Francis Group.

- Lam, C. K. C., Hoang, C. H., Lau, R. W. K., de Caux, B., Chen, Y., Tan, Q. Q., Pretorius, L., Cahusac de Caux, B., Chen, Y., Tan, Q. Q., Pretorius, L., Cahusac de Caux, B., Chen, Y., Tan, Q. Q., \& Pretorius, L. (20I9). Experiential learning in doctoral training programmes: fostering personal epistemology through collaboration. Studies in Continuing Education, 4I(I), III-I28. 
https://doi.org/ı.IO80/oI58037X.2018.I482863

- Lei, J., \& Hu, G. (2019). Doctoral candidates' dual role as student and expert scholarly writer: An activity theory perspective. English for Specific Purposes, 54, 62-74. https://doi. org/IO.IOI6/j.esp.20I8.I2.003

- Lillis, T., \& Curry, M. J. (2006). Professional Academic Writing by Multilingual Scholars. Interactions with Literacy Brokers in the Production of English-medium Texts. Written Communication, $23(\mathrm{X})$, I-33. https://doi.org/IO.II77/O74IO88305283754

- Lillis, T., \& Maybin, J. (2OI7). Introduction: The dynamics of textual trajectories in professional and workplace practice. Text and Talk, 37(4), 409-4I4. https://doi.org/IO.I5I5/text20I7-OOI7

- MacKenzie, I. S. (20I5). Writing and Publishing a Research Paper. In Human-computer Interaction. https://doi.org/Іо.ІоI6/b978-O-I2-405865-I.00008-Х

- Martínez Amilpa, D. (2018). La formación de investigadores en los programas de doctorado de la Universidad Veracruzana Doctorado Educación y Sociedad [Universitat de Barcelona]. $\quad$ https://www.tdx.cat/bitstream/handle/Io8o3/667009/DMA_TESIS. pdf? sequence $=\mathrm{I} \&$ isAllowed $=\mathrm{y}$

- Martinez, R., \& Graf, K. (20I6). Thesis Supervisors as Literacy Brokers in Brazil. Publications, $4(3)$, 26. https://doi.org/IO.339O/publications403OO26

- Matarese, V. (2OI2). Using strategic, critical reading of research papers to teach scientific writing: The reading-research-writing continuum. In Supporting Research Writing: Roles and Challenges in MultilingualSettings. Elsevier Inc. https://doi.org/IO.IOI6/B978-I-84334-666I.5OOO5-9

- Moreno Bayardo, M. G. (2007). Experiencias de formación y formadores en programas de doctorado en educación. Revista Mexicana de Investigación Educativa, I2(33), 561-580. http://www.scielo.org.mx/scielo.php?pid=SI4O5-6666200700020056I\&script=sci_arttext

- Moreno Bayardo, M. G. (2OII). La formación de investigadores como elemento para la consolidación de la investigación en la universidad. Revista de La Educación Superior, XL (2)(158), 59-78. http://www.scielo.org.mx/scielo.php?script=sci_arttext\&pid $=$ SoI85-2760201100O2000O4

- Moreno Bayardo, M. G. (2009). La aportación de los doctorados a la formación de investigadores en educación. X Congreso Nacional de Investigación Educativa, eIo. http://www.comie. org.mx/congreso/memoriaelectronica/vio/pdf/area_tematica_II/ponencias/ooo5-F.pdf

- O’Connor, M. (2003). Writing successfully in science (Second Edi). E \& FN Spon. 
- Parija, S. C., \& Kate, V. (20I7). Writing and Publishing a Scientific Research Paper. Springer. https://doi.org/IO.IOO7/978-98I-IO-4720-6

- Quiles-Fernández, E., Fernández, J. H., \& Fajardo, R. H. (2018). Formarse en la Investigación Educativa: una Comunidad de Pensamiento en Torno a la Escritura de la Tesis Doctoral. Qualitative Research in Education, 7(3), 24I-264. https://doi.org/IO.I7583/qre.20I8.3482

- Radloff, A., \& De La Harpe, B. (200o). Helping students develop their writing skills - a resource for lecturers. Flexible Learning for a Flexible Society. Higher Education Research and Development in South Asia Conference. Toowoomba, I-32.

- Rogers, E. M. (2003). Diffusion of innovations (Fifth). Free Press.

- Saeed, R., Rezaei, S., \& Seyri, H. (20I9). Iranian doctoral students' perceptions of publication in English: Motives, hurdles, and strategies. Journal of Applied Research in Higher Education, II(4), 94I-954. https://doi.org/IO.IIO8/JARHE-O2-2OI9-OO4O

- Singh, V., \& Mayer,P. (2OI4). Scientific writing: Strategies and tools for students and advisors. Biochemistry and Molecular Biology Education, 42(5), 405-4I3. https://doi.org/IO.IOO2/ bmb.208I5

-Smirnova, N. V. (20I6). Writing-for-Publication: Online Pedagogy for Post/Graduate ResearchWriting. In C. Badenhorst \& C. Guerin (Eds.), Research literacies and writing pedagogies for masters and doctoral writers (pp. 68-87). Brill Academic Publishers.

-Wisker, G. (20I5). Developing doctoral authors: engaging with theoretical perspectives through the literature review. Innovations in Education and Teaching International, 52(I), 64-74. https://doi.org/IO.IO80/I4703297.20I4.98184I

-Wortham, S. E. F. (200I). Narratives in Action: A Strategy for Research and Analysis. Teachers College Press. 